\title{
Activated breast stromal fibroblasts exhibit myoepithelial and mammary stem cells features
}

\author{
Abdelilah Aboussekhra ( $\nabla$ aboussekhra@kfshrc.edu.sa ) \\ King Faisal Specialist Hospital and Research Center https://orcid.org/0000-0001-5420-9254 \\ Syed S Islam \\ King Faisal Specialist Hospital and Research Center \\ Noura N. Alraouji \\ King Faisal Specialist Hospital and Research Center
}

Research article

Keywords: Breast cancer, cancer-associated fibroblasts, mammary stem cells, MET, myoepithelial

Posted Date: August 21st, 2020

DOI: https://doi.org/10.21203/rs.3.rs-60550/v1

License: (1) This work is licensed under a Creative Commons Attribution 4.0 International License.

Read Full License 


\section{Abstract}

Background: Active breast cancer-associated fibroblasts (CAFs) promote tumor growth and spread, and like tumor cells they are also heterogeneous with various molecular sub-types and different protumorigenic capacities.

Methods: We have used immunoblotting as well as quantitative RT-PCR to assess the expression of various epithelial/mesenchymal as well as stemness markers in breast stromal fibroblasts. Immunofluorescence was utilized to assess the level of different myoepithelial and luminal markers at the cellular level. Flow cytometry allowed to determine the proportion of CD44- and ALDH1-positive breast fibroblasts, while sphere formation assay was used to test the ability of these cells to form mammospheres.

Results: We have shown here that IL-6-dependent activation of breast and skin fibroblasts promotes mesenchymal-to-epithelial transition in a STAT3- and p16-dependent manner. Interestingly, most primary CAFs isolated from breast cancer patients exhibited such transition and expressed lower levels of the mesenchymal markers $\mathrm{N}$-cadherin and vimentin as compared to their adjacent normal fibroblasts (TCFs) isolated from the same patients. We have also shown that while CAFs and IL-6-activated fibroblasts express high levels of several myoepithelial markers such as cytokeratin $5 / 6$, cytokeratin 14 , cytokeratin 19 and c-KIT, they do not express the luminal marker cytokeratin 18. Furthermore, we present clear

evidence that IL-6 treatment promotes mammary stemness characteristics (CD44 high/CD2 $4^{\text {low }} / A L D H^{\text {high }}$ ) in stromal fibroblasts in a STAT3- and p16-dependent manner. Similar features were also identified in 12 CAFs isolated from breast tumors, which showed higher proportions of CD44 $4^{\text {high }}$ and ALDH ${ }^{\text {high }}$ cells, compared to their corresponding TCF cells. Importantly, these CD44high cells have higher abilities to form mammospheres and to enhance cell proliferation of breast cancer cells in a paracrine manner relative to their corresponding CD $44^{\text {low }}$ cells.

Conclusions: Together, the present findings show novel characteristics of active breast stromal fibroblasts, which exhibit additional myoepithelial/progenitor features.

\section{Background}

Breast cancer-associated fibroblasts (CAFs) have different origins, and while some remain quiescent the majority are active with cancer-promoting capacity, and are also referred to as myofibroblasts $[1,2]$. While fibroblasts exhibit a thin and small spindle morphology, active CAFs are large and flat cells with different shapes and prominent nucleoli [3]. Owing to lack of precise molecular definition of CAFs, these cells are most often defined by their morphological characteristics and the expression of some markers [4]. The pro-carcinogenic effects of myofibroblasts are related to the production of high levels of growth factors, cytokines and chemokines, which can promote epithelial-to-mesenchymal transition (EMT) as well as stemness [5-7]. Although most of these cells express high levels of a-SMA, active fibroblasts constitute a large and heterogeneous cell pool expressing different molecular markers, which are not specific for 
fibroblasts, such as fibroblast activation protein (FAP) and fibroblast-specific protein-1 (FSP-1) [2, 8]. In a recent study, Su et al. have identified a subset of active CAF cells, which express CD10 and GPR77 and can promote stemness and chemoresistance within breast cancer cells [9]. Therefore, what are CAFs ? In fact, these myofibroblasts share several molecular and cellular features with normal fibroblasts, their shape and secretome resemble those of senescent cells, express some myoepithelial markers and exhibit some cancer features $[2,4,10]$. These findings raised an important question on the identity of these cells.

Aside from promoting breast carcinogenesis, active fibroblasts can modulate cancer response, drug accessibility and immune regulation, which explains their association with poor patient survival [11]. Thereby, myofibroblasts have gained momentum in anticancer drug discovery, taking into account their genetic stability and their presence across different cancer types [11]. To achieve this, better characterization of these cells and their functional interplay with cancer cells is mandatory. We have recently shown using indirect co-culturing that triple-negative breast cancer cells can increase the expression of the a-SMA protein and activate breast stromal fibroblasts in an IL-6-dependent manner. This also enhanced their migratory/invasiveness as well as their proliferative capacities [12]. Therefore, we sought in the present study to further characterize these active CAFs at both the molecular and cellular levels. We have shown that the activation of breast stromal fibroblasts triggers mesenchymal-toepithelial transition, and also promotes the expression of stemness markers.

\section{Materials And Methods Cells and cell culture}

Breast fibroblast cells were obtained and used as previously described [10]. MCF-7 and MDA-MB-231 cells were purchased from ATCC and were authenticated using short tandem repeat profiling by ATCC, propagated, expanded, and frozen immediately into numerous aliquots after arrival. Cells were regularly screened for mycoplasma contamination using MycoAlert Mycoplasma Detection Kits (Lonza). All supplements were obtained from Sigma (Saint Louis, MO, USA) except for antibiotic and antimycotic solutions, which were obtained from Gibco (Grand Island, NY, USA). Cells were maintained at $37^{\circ} \mathrm{C}$ in humidified incubator with $5 \% \mathrm{CO}_{2}$.

Human IL-6 recombinant protein (hBA-184) (Santa Cruz, CA).

\section{Cellular lysate preparation and immunoblotting}

This has been performed as previously described (5). Antibodies directed against, Twist1 (10E4E6), aSMA, IL-6, Snail1 (C15D3) and Vimentin (RV202) were purchased from Abcam (Cambridge, MA). ALDH1/2 (H-85), CD24 (C-20), and GAPDH (FL-335) were purchased from Santa Cruz Biotech (Santa Cruz, CA). p16 was purchased from BD. E-cadherin (24E10), N-Cadherin, AKT (C73H10), p-AKT (Thr308), Oct-4 (C30A3), Sox2 (D6D9) and EpCAM (D1B3) Cell Signaling Technology (Danvers, MA). CD44 was purchased from (Sigma-Aldrich). 


\section{RNA purification and qRT-PCR}

Total RNA was purified using the miRNeasy mini kit (Qiagen, USA) according to the manufacturer's instructions and was treated with RNase-free DNase. RNA $(1 \mu \mathrm{g})$ was used to synthesize complementary deoxyribonucleic acid (cDNA) utilizing the Advantage RT-PCR kit. qRT-PCR was performed in triplicate using cDNA $(4 \mu \mathrm{l})$ mixed with $2 x$ FastStart Essential DNA Green qPCR master mix ( $5 \mu \mathrm{l})$ and primers mix $(1 \mu \mathrm{l})$. Amplifications were performed utilizing the LightCycler 96 Real-time PCR detection system. GAPDH expression levels were used for normalization, and gene expression differences were calculated using the threshold cycle (Ct). The respective primers are:

GAPDH: 5'-GAGTCCACTGGCGTCTTC-3' and 5'-GGGGTGCTAAGCAGTTGGT-3'

CDH2: 5'-CCT CCA GAG TTT ACT GCC ATG AC-3' and 5'-GTA GGA TCT CCG CCA CTG ATT C-3'

CDH1: 5'-CCC GCC TTA TGA TTC TCT GCT CGT G-3' and 5'-TCC GTA CAT GTC AGC CAG CTT CTT G-3'

ALDH1A1: 5'-TCT CTA TTT CTC TCC CCT CCC T-3' and 5'ACC ATC TTT GAA GGG TTG GC-3'

siRNA transfection

STAT3 siRNA and control siRNA were obtained from Cell Signaling technology. The transfections were carried out using the High Perfect reagent (Qiagen, USA) as recommended by the manufacturer.

\section{CDKN2A-ORF transfection}

Lentivirus-based vectors bearing CDKN2A-ORF and the corresponding control were used to carry out transfections using human dermal fibroblast nucleofector 2000 transfection kit (Invitrogen, USA) following the manufacturer's recommendations. After 5 days, transfected cells were selected with puromycin $(1 \mu \mathrm{g} / \mathrm{mL})$.

\section{Flow cytometry}

Cells were incubated for 30 min on ice with directly labeled antibodies. Cells were then washed with PBS followed by fixation in $0.5 \%$ paraformaldehyde in PBS. Data were acquired using an LSR II flow cytometer using BD FACSDiva operating software. Positive staining was considered based on the negativity of an isotype control. A minimum of 10,000 events were recorded for all samples.

\section{Cell sorting, flow cytometry and Aldeflour assay}

CD44 + and CD44- cells were selected from different sets of CAFs by flow cytometric cell sorting (FACS). The FACS was performed on single cell suspensions using the flow cytometer (BD FACSAria). Before FACS sorting, single CAF cells were resuspended in PBS containing 1\% FBS and incubated with antibody against CD44 for 30 min at $4{ }^{\circ} \mathrm{C}$. For CD44 flow cytometry analysis single cells were resuspended in PBS containing $1 \%$ PBS and werd stained with fluorescent-conjugated antibody against CD44. Aldeflour assay 
was performed according to manufacturer's instruction (StemCell Technology). Flow cytometry data were acquired using NovoCyte Flow Cytometer (ACEA Bioscience).

\section{Immunofluorescence}

Cells for immunofluorescence were blocked with $4 \%$ paraformaldehyde for $15 \mathrm{~min}$ at room temperature, washed with PBS and permeabilized with $0.25 \%$ Triton X-100 in PBS for $10 \mathrm{~min}$. After washing with PBS, cells were incubated with $4 \%$ BSA for 30 min, incubated with indicated primary antibodies overnight at $4{ }^{\circ} \mathrm{C}$. Washed with PBS and incubated with Alexa Fluor conjugated secondary antibodies for $30 \mathrm{~min}$. Next, cells were counter stained with Alexa Flour 488 Phalloidin for cytoskeleton and DAPI for nuclei. Images were captured by Olympus microscope. Primary antibodies used: Cytokeratin 14 (LL002), Cytokeratin 19 (EP1580Y), Cytokeratin 18, Cytokeratin 5/6, and c-Kit were purchased from Abcam.

\section{Sphere formation assay}

FACS sorted CD44+/CD44- CAF cells (5000 cells/ml) were cultured in ultra-low attachment plates (Corning) in serum-free DMEM-F12 (Life Technologies) containing stem cell culture supplements. After 10 days in culture, spheres $>50 \mu \mathrm{m}$ were counted. Three independent experiments were conducted.

\section{Coculture experiments}

Co-culture experiments were performed by seeding MCF-7 breast cancer cells $\left(5.10^{4}\right)$ in the lower chamber and FACS sorted CD44+/CD44- CAF cells $\left(5.10^{4}\right)$ in the upper chamber of 6-well transwell apparatus with $0.4 \mu \mathrm{m}$ pore (Corning NY, USA), and were incubated for $48 \mathrm{~h}$. MCF-7 cells were trypsinized and counted.

\section{Cell migration, invasion and proliferation assays}

This has been performed as previously described [13]. These assays were performed in a label-free realtime setting using the XCELLigence RTCA technology (Roche, Germany) that measures impedance changes in a meshwork of interdigitated gold microelectrodes located at the well bottom (E-plate) or at the bottom side of a micro-porous membrane (CIM plate 16). Cell migration and invasion were assessed as per the manufacturer's instructions. In brief, $2 \times 10^{4}$ cells in serum-free medium were added to the upper wells of the CIM-plate coated with a thin layer of Matrigel (BD Biosciences) basement membrane matrix diluted 1:20 in serum-free medium (invasion) or non-coated (migration). Complete medium was used as a chemo-attractant in the lower chambers. Subsequently, the plates were incubated in the RTCA for $24 \mathrm{~h}$ and the impedance value of each well was automatically monitored by the xCELLigence system and expressed as Cell Index $(\mathrm{Cl})$ value, which represents cell status based on the measured electrical impedance change divided by a background value. Each assay was biologically performed in triplicate.

For the proliferation assay, exponentially growing cells $\left(2 \times 10^{4}\right)$ were seeded in E-plate with complete medium as per the manufacturer's instruction. Cell proliferation was assessed for $48 \mathrm{~h}$. All data were recorded and analyzed by the RTCA software. Cell Index was used to measure the change in the electrical 
impedance divided by background value, which represents cell status. Each assay was biologically performed in triplicate.

\section{Conditioned media}

Cells were cultured in medium without serum for $24 \mathrm{~h}$, and then media were collected and briefly centrifuged. The resulting supernatants were used either immediately or were frozen at $-80^{\circ} \mathrm{C}$ until needed.

\section{Results}

\section{Activation of human fibroblasts promotes mesenchymal-to- epithelial transition}

We have previously shown that serum-free medium (SFM) conditioned with MDA-MB-231 cells (MDASFCM) and the interleukin-6 activates breast stromal fibroblasts [12]. To confirm this, whole cell lysates were prepared form normal breast fibroblast (NBF-6) cells exposed either to IL-6 $(3.5 \mathrm{ng} / \mathrm{mL})$ or SFCM from MDA-MB-231 cells treated either with IL-6 specific siRNA or a scramled sequence used as control. SFM was used as negative control. Figure 1A shows IL-6- and MDA-SFCM-dependent up-regulation of the myofibroblast marker a-SMA, and this effect was abolished in cells wherein IL- 6 was knocked-down as compared to controls. Next, we investigated the possible changes in the expression of the epithelial and/or mesenchymal markers. Figure 1A shows IL-6-dependent increase in the epithelial markers (Ecadherin and EpCAM), and a decrease in the mesenchymal markers (N-cadherin and vimentin). Similarly, MDA-SFCM and IL-6 up-regulated a-SMA as well as the epithelial markers (E-cadherin and EpCAM), while reduced the level of the mesenchymal markers (N-cadherin and vimentin) in the human skin fibroblasts HFSN1 (Fig. 1B). In addition, the migration/invasion as well as proliferation of HFSN1 cells were higher in cells treated with IL-6 or MDA-SFCM as compared to SFM (Fig. 1C). This was confirmed at the molecular level by showing AKT activation upon treatment with IL-6 or MDA-SFCM (Fig. 1D). This indicates the induction of the mesenchumal-to-epithelial transition (MET) upon activation of fibroblast cells.

\section{IL-6 induces MET features in breast stromal fibroblasts in a STAT3- and p16-dependent manner}

We have previously shown that myofibroblasts express low levels of p16, and IL-6-dependent activation of breast stromal fibroblasts is inhibited by p16 up-regulation or STAT3 knock-down [12, 14]. Therefore, we sought to investigate the effect of these genes in IL-6-dependent induction of the MET features in breast stromal fibroblasts (NBF-6). Figure 1E shows that ectopic expression of p16 inhibits IL-6-related up-regulation of a-SMA and also the MET-related features as compared to control cells. Indeed, p16 upregulation abolished IL-6-dependent increase in the level of the epithelial markers (E-cadherin and EpCAM) as well as the decrease in the level of the mesenchymal markers ( $\mathrm{N}$-cadherin and vimentin) 
compared to controls (Fig. 1E). Furthermore, p16 abolished IL-6-dependent activation of AKT (Fig. 1E). Interestingly, similar results were obtained when STAT3 was knocked-down by specific siRNA (Fig. 1F). This indicates that p16 down-regulation and STAT3 activation are important for IL-6-dependent induction of the MET process in breast fibroblasts.

\section{Breast cancer-associated fibroblasts display mesenchymal- to-epithelial transition}

To confirm that active breast stromal fibroblasts express high levels of the epithelial markers and low levels of the mesenchymal ones, we assessed the expression of some key markers of the MET process in 12 pairs CAF/TCF (tumor counterpart fibroblasts) isolated from 12 breast cancer patients. To this end, cell lysates were prepared from these cells and were used for immunoblotting analysis using specific antibodies. As expected, most CAFs (8 out of 12) expressed higher levels of a-SMA than their corresponding TCFs (Fig. 2A). Interestingly, all CAFs expressed higher level of E-cadherin and lower levels of the mesenchymal markers $\mathrm{N}$-cadherin and vimentin as compared to their corresponding TCFs (Fig. 2A). The decrease in the expression of the major mesenchymal marker $\mathrm{N}$-cadherin and the upregulation of the major epithelial marker E-cadherin in CAF cells was confirmed at the mRNA level by quantitative reverse transcription-PCR (qRT-PCR). Indeed, the $C D H 2$ mRNA level was significantly reduced, while $C D H 1$ mRNA level was significantly induced in $6 \mathrm{CAFs}$ as compared to their corresponding TCFs (Fig. 2B and C). This confirms that the MET process was induced in CAFs isolated from breast cancer patients.

\section{Breast myofibroblast cells exhibit myoepithelial features}

The fact that active CAFs express high levels of a-SMA and also exhibit epithelial characteristics, prompted us to investigate whether they gained myoepithelial features, since these cells also express aSMA. Therefore, we assessed the expression of the main myoepithelial markers cytokeratin 14 and cytokeratin 19 in CAF/TCF pairs as well as in normal breast fibroblast cells (NBF-6) by immunofluorescence. Figure 3A shows that NBF- 6 cells express very low levels of both cytokeratins 14 and 19, which were up-regulated in the active IL- 6 treated cells. Similarly, the active CAF-64 and CAF-180 cells expressed higher levels of cytokeratin 14 and cytokeratin 19 relative to their correspondent adjacent counterparts TCF-64 and TCF-180, respectively (Fig. 3A). In a double immunostaining experiment, it was confirmed the expression of cytokeratin 14 and cytokeratin 19 but not the luminal specific cytokeratin 18 in CAF-64 cells (Fig. 3B). However, the three cytokeratins were almost undetectable in the corresponding TCF-64 cells (Fig. 3B). Similar results were obtained for CAF/TCF-900 pair (Fig. 3C). To confirm the acquisition of epithelial features by CAF cells, we have also shown that CAF-900 cells, but not their TCF900 counterpart, express 2 other myoepithelial markers c-Kit and cytokeratin 5/6 (Fig. 3D and E). Together, these results indicate that active breast fibroblasts acquired myoepithelial features. 


\section{IL-6 induces stemness features in fibroblast cells in a STAT3- and p16-dependent manner}

Since the MET process is associated with the induction of stemness and pluripotency in fibroblasts [1517], and mammary stem cells exhibit myoepithelial cell properties [18], we sought to assess the expression of some important stemness markers, and have found that treatment with IL-6 or MDA-SFCM induced mammary stem cells features (CD44 $\left.{ }^{\text {high }} / \mathrm{CD} 24^{\text {low }} / A L D H^{\text {high }}\right)$ in both NBF-6 as well as HFSN1 fibroblasts (Fig. 4A and B). This suggests that myofibroblasts or CAFs exhibit epithelial as well as stemness features. Figure $3 \mathrm{C}$ shows that ectopic expression of p16 inhibits IL-6-related up-regulation of ALDH/CD44 and down-regulation of CD24. Similar effect was obtained with STAT3 specific siRNA (Fig. 4D). This shows that, like MET, IL-6-dependent induction of stemness features in breast stromal fibroblasts requires STAT3 induction and p16 down-regulation.

\section{CAFs from breast cancer tissues exhibit stemness features}

Next, we have checked the status of the stemness markers in CAF/TCF pairs isolated from breast cancer tissues. Flow cytometry analysis shows clear increase in the proportion of CD44 ${ }^{\text {high }}$ subpopulation in CAF cells (CAF-64, CAF-87 and CAF-900 with 7,59\%, 7.8\% and $4.73 \%$, respectively) as compared to their corresponding counterparts (TCF-64, TCF- 87 and TCF-900 with $1.69 \%, 2.05 \%$ and $1.24 \%$, respectively) (Fig. 5A). Additionally, we examined the ALDH (aldehyde dehydrogenase) activity in paired CAFs and TCFs cells. We used gating strategy for the exclusion by incubating cells with DEAB and were used as negative control (Fig. 5B). Remarkably, all CAF cells (CAF-64, CAF-87 and CAF-900) exhibited higher ALDH activity $(57.21 \%, 21.31 \%$ and $5.3 \%$ ) as compared to their corresponding TCF cells (TCF-64, TCF-87 and TCF-900) with ALDH activity as low as $37.89 \%, 14.27 \%$ and $1.52 \%$, respectively (Fig. 5B).

Furthermore, Fig. $5 \mathrm{C}$ shows that most CAFs express the markers of mammary stem cells (CD44 $4^{\text {high }} / \mathrm{CD} 24^{\text {low }} / A L D H^{\text {high }}$ ) as compared to their corresponding TCF cells. Indeed, relative to their corresponding TCFs, 7 out of 12 CAFs expressed higher level of ALDH1, 9 out of 12 expressed higher level of CD44, and 10 out of 12 showed lower level of CD24 (Fig. 5C). Interestingly, 8 out 12 CAFs showed the CD $44^{\text {high }} / \mathrm{CD} 24^{\text {low }}$ characteristic, and 6 out of 12 CAFs exhibited the (CD44 high $\left./ C D 24^{\text {low }} / A L D H^{\text {high }}\right)$ feature of mammary stem cells (Fig. 5C). The increase in the expression level of ALDH1 was confirmed at the mRNA level, by showing that 6 CAFs expressed higher levels of the ALDH1 mRNA compared to their corresponding TCFs (Fig. 5D). On the other hand, the pluripotency marker Sox-2 was very lowly expressed in the majority of the CAF/TCF pairs, and showed only a slight increase in CAF-64, CAF-153 and CAF-69 cells relative to their corresponding TCFs (Fig. 5C). These results indicate that active fibroblasts show MET as well as stemness features but they did not reach pluripotency. 


\section{CD44-positive CAF cells have higher ability to form spheres and enhance breast cancer cell growth in vitro}

After showing that a proportion of CD44-positive CAF cells express mammary stem cells markers and exhibit higher ALDH activity, we sought to investigate the potential of these cells in forming mammospheres. To this end, we have first sorted CD44 high cells from various CAF cells, and then the sorted cells were used for sphere formation in ultralow attachment plates. Interestingly, CD $44^{\text {high }}$ cells generated significantly more spheres than CD44 ${ }^{\text {low }}$ cells from all three CAF cultures (Fig. 6A). Next, we investigated the paracrine pro-carcinogenic effect of these CAF-CD $44^{\text {high }}$ cells, by investigating their effect on the proliferation of MCF-7 cells in a coculture setting. Therefore, we isolated CD44 ${ }^{\text {high }}$ and CD44 ${ }^{\text {low }}$ cells from 3 different CAF cells as described above and cocultured them separately with MCF-7 cells. Figure 6B shows clear differences in MCF-7 cell growth between CD44 ${ }^{\text {low }}$ and their corresponding CD44 ${ }^{\text {high }}$ cells. Indeed, MCF-7 growth was significantly enhanced in the presence of CD $44^{\text {high }}$ cells as compared to their corresponding CD44 ${ }^{\text {low }}$ cells (Fig. 6B). These results suggest that CAF cells contain CD44 ${ }^{\text {high }}$ subpopulation with stem cell features and higher paracrine pro-carcinogenic effects.

\section{Discussion}

Cancer-associated fibroblasts are very active promoters of breast carcinogenesis through functional cross-talks with cancer cells via secretions that contain metabolites, cytokines and other types of molecules. CAFs constitute a large heterogeneous population of cells that express various types of markers, which are not unique for these cells. This vast heterogeneity could be owing to various origins of CAFs or their mode/condition of activation [2, 4]. We have shown in the present study that MDA-MB-231and IL-6-dependent activation of normal fibroblasts upregulates the epithelial markers (E-cadherin and EpCAM) but downregulates the mesenchymal markers ( $\mathrm{N}$-cadherin and vimentin). This suggests that the activation of fibroblasts is accompanied by a transition from the mesenchymal to the epithelial state (MET). Intriguingly, despite this MET, these cells showed higher migration, invasion and proliferation capacities as compared to controls (Fig. 1C and [12]). This could be explained by the fact that these cells express high levels of the active form of the pro-invasive/migratory and -proliferative protein kinase AKT (Fig. 1D). Interestingly, ectopic expression of p16 or knock-down of STAT3 in breast stromal fibroblasts suppressed the IL-6-related activation of these cells and their transition to epithelial status. This indicates that while p16 inhibits the IL-6 induced MET, STAT3 activation was required for the acquisition of the epithelial traits.

The development of epithelial features and down-regulation of mesenchymal markers were confirmed in 12 patient-derived CAFs and their adjacent counterpart TCFs. The active status of CAFs was confirmed by the higher expression level of a-SMA, which was accompanied in most CAFs with increased level of Ecadherin and reduced level of $\mathrm{N}$-cadherin and vimentin as compared to their corresponding TCFs. This 
indicates that, like MDA-MB-231 cells and IL-6, breast cancer cells activate fibroblasts and trigger MET within tumors. It has been previously shown that the complete reprogramming of fibroblasts into pluripotent stem cells entails also up-regulation of the epithelial markers and down-regulation of the mesenchymal markers [15-17]. This confirms the importance of the MET process during fibroblast reprogramming.

Importantly, active CAFs expressed high levels of the myoepithelial markers cytokeratin 14, cytokeratin 19 , cytokeratin $5 / 6$ as well as c-Kit, in addition to a-SMA, which is highly expressed in both myofibroblasts and myoepithelial cells. However, CAFs did not express cytokeratin 18 , the luminal cells biomarker. This indicates that active CAFs acquired myoepithelal cells characteristics. These findings confirm what has been recently shown by Su et al. who have shown the expression of the myoepithelial cells marker CD10 on a subset of active CAF cells [9]. This suggests that active fibroblasts develop myo-fibroblast as well as myo-epithelial traits, with a novel shape different from that of fibroblast and myoepithelial cells. Indeed, while myoepithelial cells are stellate in shape, and fibroblasts have spindle-shape, myofibroblasts are characterized by branching and flat morphology $[2,4]$.

Since a-SMA-expressing myoepithelial cells were shown to exhibit some features of lineage-restricted stem cells [18], we checked this possibility in active breast fibroblasts. We have shown that IL-6-activated breast and skin fibroblasts also exhibited the (CD44 high $\left./ C D 24^{\text {low }} / A L D H^{\text {high }}\right)$ feature of mammary stem cells, in a p16- and STAT3-dependent manner. In addition, we have shown that the proportions of CD44 ${ }^{\text {high }}$ as well as ALDH ${ }^{\text {high }}$ sub-populations were higher in CAF cells compared to their counterpart TCF cells isolated from the same patient. This showed that activated fibroblasts acquired stem/progenitor cell features. This was confirmed in 12 pairs CAF/TCF cells isolated from 12 different patients. Indeed, most of these cells exhibited the (CD44 ${ }^{\text {high }} / \mathrm{CD} 24^{\text {low }} / \mathrm{ALDH}^{\text {high }}$ ) feature of mammary stem cells (Fig. 5C). Together, these findings indicate that CAFs are myofibroblasts that express myoepithelial as well as stem cell markers, with high migratory/invasive and proliferative capacities. This confirms that they do not represent a cell type but rather a cell state, as has been previously proposed by Madar et al. [19].

Interestingly, we have also shown that this $\mathrm{CD} 44^{\text {high }}$ sub-population of CAF cells has much higher capacity to grow in suspension and form mammospheres compared to CD44 low sub-population. Furthermore, these cells have higher capacity to promote cell growth of breast cancer cells in a co-culture setting (Fig. 5). Similarly, it has been previously shown that CD44 was abundantly expressed on immortalized CAF cells isolated from melanoma bearing mice, and that CD44-positive cells supported the stemness and drug resistance of cancer cells [20]. This indicates that a proportion of CAFs exhibit stemness features and have higher paracrine pro-carcinogenic effects than the corresponding CD44negative cells. The fact that the pluripotency marker Sox-2 was only lowly expressed in most CAF/TCF pairs, indicates that these CAF-stem cells did not reach pluripotency. Thereby, which type of stem cells are formed in active breast fibroblasts? and what are their origin ? Ishikawa et al. have shown simultaneous expression of cancer stem cell-like and CAF-like properties in a primary culture of breast cancer cells [21]. In another study, it has been reported the presence of multipotent CAF cells in hepatocellular carcinoma 
tissues [22]. Furthermore, Herrera et al showed that most pro-migratory CAFs from human colon tumors expressed stem cell markers [23]. Together, these findings indicate that active CAFs contain a subpopulation of cells with stem features, and that this population possesses the higher pro-carcinogenic ability. In a recent publication, Nair et al. have proposed a cancer stem cell model as the point of origin of CAFs [24]. This suggests that CAF cells in a tumor may have different origins with different gene expression signature and consequently different effects on cancer cells. It's also possible that CAF heterogeneity arises from different paracrine signaling effects from heterogeneous cancer cells as well as other active stromal cells such as adipocytes and macrophages. Indeed, we have shown here that TNBC cells (MDA-MB-231) as well as IL-6 can promote stemness in breast stromal fibroblasts.

\section{Conclusions}

Together, these results indicate that great proportion of active breast stromal fibroblasts develop myoepithelial/progenitor cells features. This allows a better understanding of these cells, which represent a permanent cell state rather than a cell type, and opens new avenues towards their specific therapeutic targeting.

\section{Abbreviations}

BSF, breast stromal fibroblast; CAF, cancer-associated fibroblast; IL-6, interleukin-6; SFM, Serum-free media; SFCM, Serum-free conditioned media; TCF, tumor counterpart fibroblast;

\section{Declarations}

\section{Ethics approval and consent to participate}

Written informed consent was not required and a waiver was granted since the study was retrospective and samples were anonymized to the research team. This was granted by the institutional review board approval (RAC\#2180018).

\section{Consent of publication}

Not applicable

\section{Availability of data and material}

The data generated, used and analyzed in the current study are available from the corresponding author in response to reasonable request.

\section{Competing interests}


The authors declare that they have no potential financial competing interest.

\section{Funding}

This work was solely supported by the King Faisal Specialist Hospital and Research Centre

\section{Author's contributions}

Conception and design: Abdelilah Aboussekhra; Development of methodology and acquisition of data: Noura Alraouji, Syed Islam, Analysis and interpretation of data: Noura Alraouji, Syed Islam, Abdelilah Aboussekhra; Writing of the manuscript: Noura Alraouji, Syed Islam, Abdelilah Aboussekhra.

\section{Acknowledgments}

We are thankful to Mrs. Fauziah Hendrayani for her technical support. This work was performed under the RAC proposal \# 2180018.

\section{References}

1. Kalluri R. The biology and function of fibroblasts in cancer. Nat Rev Cancer. 2016;16(9):582-98.

2. Sahai E, Astsaturov I, Cukierman E, DeNardo DG, Egeblad M, Evans RM, et al. A framework for advancing our understanding of cancer-associated fibroblasts. Nat Rev Cancer. 2020;20(3):174-86.

3. Kalluri R, Zeisberg M. Fibroblasts in cancer. Nat Rev Cancer. 2006;6(5):392-401.

4. Gascard P, TIsty TD. Carcinoma-associated fibroblasts: orchestrating the composition of malignancy. Genes Dev. 2016;30(9):1002-19.

5. Huang TX, Guan XY, Fu L. Therapeutic targeting of the crosstalk between cancer-associated fibroblasts and cancer stem cells. Am J Cancer Res. 2019;9(9):1889-904.

6. Chan TS, Shaked Y, Tsai KK. Targeting the Interplay Between Cancer Fibroblasts, Mesenchymal Stem Cells, and Cancer Stem Cells in Desmoplastic Cancers. Front Oncol. 2019;9:688.

7. Fiori ME, Di Franco S, Villanova L, Bianca P, Stassi G, De Maria R. Cancer-associated fibroblasts as abettors of tumor progression at the crossroads of EMT and therapy resistance. Mol Cancer. 2019;18(1):70.

8. Buchsbaum RJ, Oh SY. Breast Cancer-Associated Fibroblasts: Where We Are and Where We Need to Go. Cancers. 2016; 8(2).

9. Su S, Chen J, Yao H, Liu J, Yu S, Lao L, et al. CD10(+)GPR77(+) Cancer-Associated Fibroblasts Promote Cancer Formation and Chemoresistance by Sustaining Cancer Stemness. Cell. 2018;172(4):841-56. e16. 
10. Hawsawi NM, Ghebeh H, Hendrayani SF, Tulbah A, Al-Eid M, Al-Tweigeri T, et al. Breast carcinomaassociated fibroblasts and their counterparts display neoplastic-specific changes. Cancer Res. 2008;68(8):2717-25.

11. Chen X, Song E. Turning foes to friends: targeting cancer-associated fibroblasts. Nat Rev Drug Discov. 2018;18:99-115.

12. Hendrayani SF, Al-Khalaf HH, Aboussekhra A. The Cytokine IL-6 Reactivates Breast Stromal Fibroblasts through Transcription Factor STAT3-dependent Up-regulation of the RNA-binding Protein AUF1. J Biol Chem. 2014;289(45):30962-76.

13. Silva G, Aboussekhra A. p16(INK4A) inhibits the pro-metastatic potentials of osteosarcoma cells through targeting the ERK pathway and TGF-beta1. Mol Carcinog. 2016;55(5):525-36.

14. Al-Ansari MM, Hendrayani SF, Shehata Al, Aboussekhra A. p16(INK4A) Represses the paracrine tumor-promoting effects of breast stromal fibroblasts. Oncogene. 2013;32(18):2356-64.

15. Li R, Liang J, Ni S, Zhou T, Qing X, Li H, et al. A mesenchymal-to-epithelial transition initiates and is required for the nuclear reprogramming of mouse fibroblasts. Cell Stem Cell. 2010;7(1):51-63.

16. Liu X, Sun H, Qi J, Wang L, He S, Liu J, et al. Sequential introduction of reprogramming factors reveals a time-sensitive requirement for individual factors and a sequential EMT-MET mechanism for optimal reprogramming. Nat Cell Biol. 2013;15(7):829-38.

17. Samavarchi-Tehrani P, Golipour A, David L, Sung HK, Beyer TA, Datti A, Woltjen K, Nagy A, Wrana JL. Functional genomics reveals a BMP-driven mesenchymal-to-epithelial transition in the initiation of somatic cell reprogramming. Cell Stem Cell. 2010;7(1):64-77.

18. Prater MD, Petit V, Alasdair Russell I, Giraddi RR, Shehata M, Menon S, et al. Mammary stem cells have myoepithelial cell properties. Nat Cell Biol. 2014;16(10):942-50. 1-7.

19. Madar S, Goldstein I, Rotter V. 'Cancer associated fibroblasts'-more than meets the eye. Trends Mol Med. 2013;19(8):447-53.

20. Kinugasa Y, Matsui T, Takakura N. CD44 expressed on cancer-associated fibroblasts is a functional molecule supporting the stemness and drug resistance of malignant cancer cells in the tumor microenvironment. Stem Cells. 2014;32(1):145-56.

21. Ishikawa M, Inoue T, Shirai T, Takamatsu K, Kunihiro S, Ishii H, Nishikata T. Simultaneous expression of cancer stem cell-like properties and cancer-associated fibroblast-like properties in a primary culture of breast cancer cells. Cancers. 2014;6(3):1570-8.

22. Sukowati $\mathrm{CH}$, Anfuso B, Croce LS, Tiribelli C. The role of multipotent cancer associated fibroblasts in hepatocarcinogenesis. BMC Cancer. 2015;15:188.

23. Herrera M, Islam AB, Herrera A, Martin P, Garcia V, Silva J, et al. Functional heterogeneity of cancerassociated fibroblasts from human colon tumors shows specific prognostic gene expression signature. Clin Cancer Res. 2013;19(21):5914-26.

24. Nair N, Calle AS, Zahra MH, Prieto-Vila M, Oo AKK, Hurley L, et al. A cancer stem cell model as the point of origin of cancer-associated fibroblasts in tumor microenvironment. Sci Rep. 2017;7(1):6838. 


\section{Figures}

Figure 1

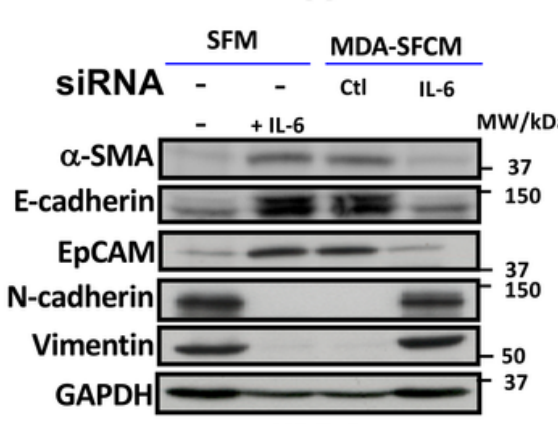

NBF-6
B

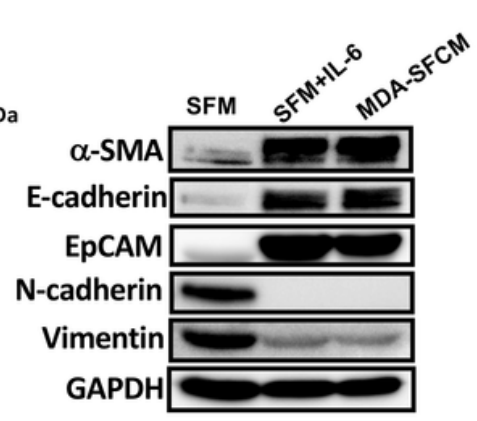

HFSN-1

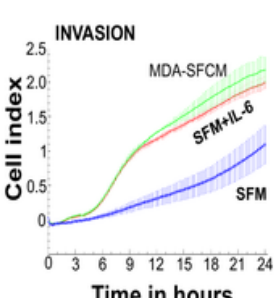

Time in hours

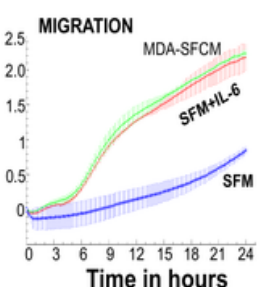

HFSN1

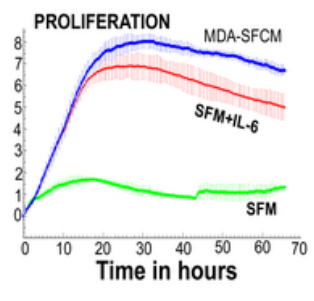

HFSN1

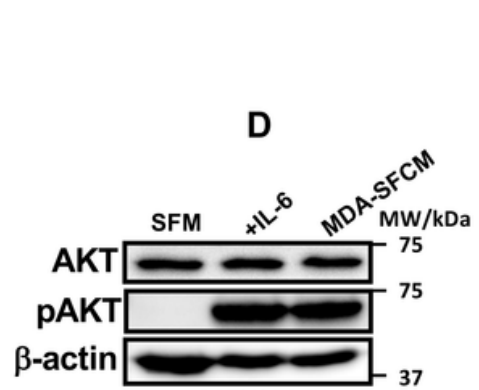

HFSN1
F

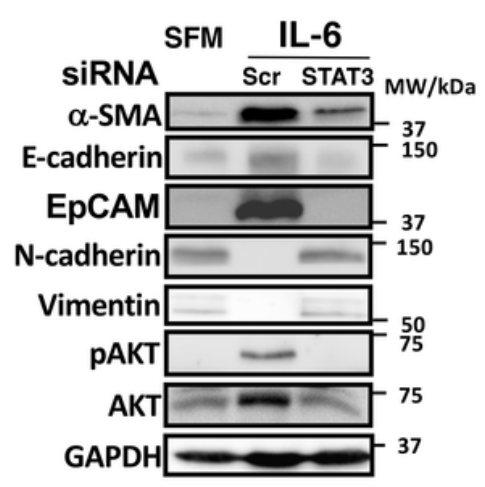

\section{Figure 1}

IL-6 induces MET features in human fibroblast cells. (A and B) Whole cell lysates were prepared from the indicated cells treated as shown, and then immunoblotting analysis was performed using antibodies against the indicated proteins. GAPDH was used as internal control. C, Exponentially growing HFSN1 cells were treated as indicated, and then the proliferation, migration and invasion capabilities were assessed using the RTCA-DP xCELLigence system. D, Whole cell lysates were prepared from the indicated cells treated as shown, and then immunoblotting analysis was performed. E, p16 was ectopically expressed in NBF-6 and an empty vector was used as control (Ctl). Cells were then exposed to SFM or SFM containing IL-6 as indicated. Subsequently, whole cell lysates were prepared, and then immunoblotting analysis was performed using specific antibodies against the indicated proteins. $F$, STAT3 was knocked-down in NBF-6 cells using specific siRNA (a scrambled sequence was used as control, Scr). Whole cell lysates were prepared, and then immunoblotting analysis was performed using specific antibodies against the indicated proteins. 
Figure 2

A

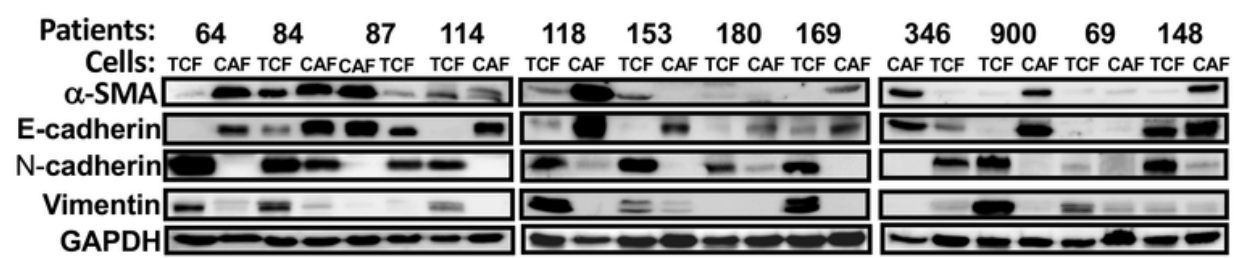

B

C

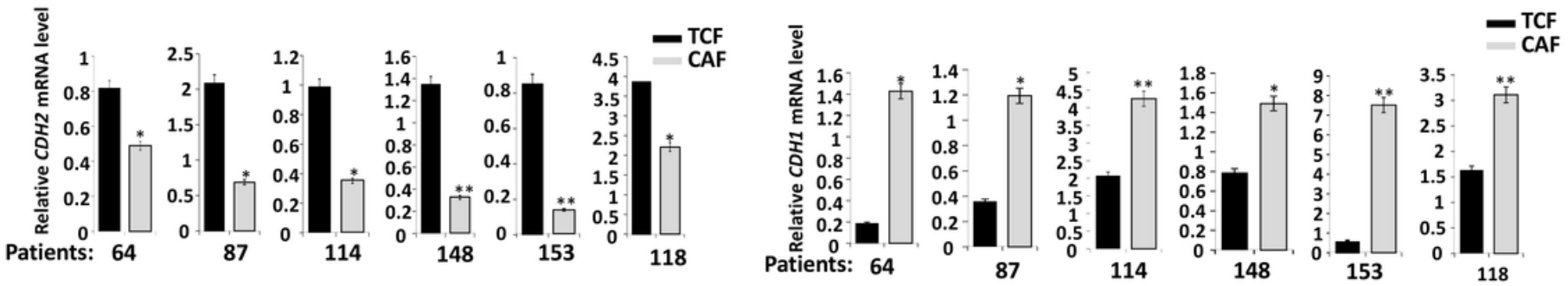

Figure 2

Cancer-associated fibroblasts express low levels of mesenchymal markers. A, Whole cell lysates were prepared from the indicated cells and the expression levels of the indicated proteins were assessed by immunoblotting. B and C, Total RNA was extracted from the indicated cells, and then the mRNA levels of the indicated genes were determined by qRT-PCR. Error bars indicate mean $\pm S D,(n=3) . *$ : $p \leq 0.05 ; * \star p$ $\leq 0.01$. 

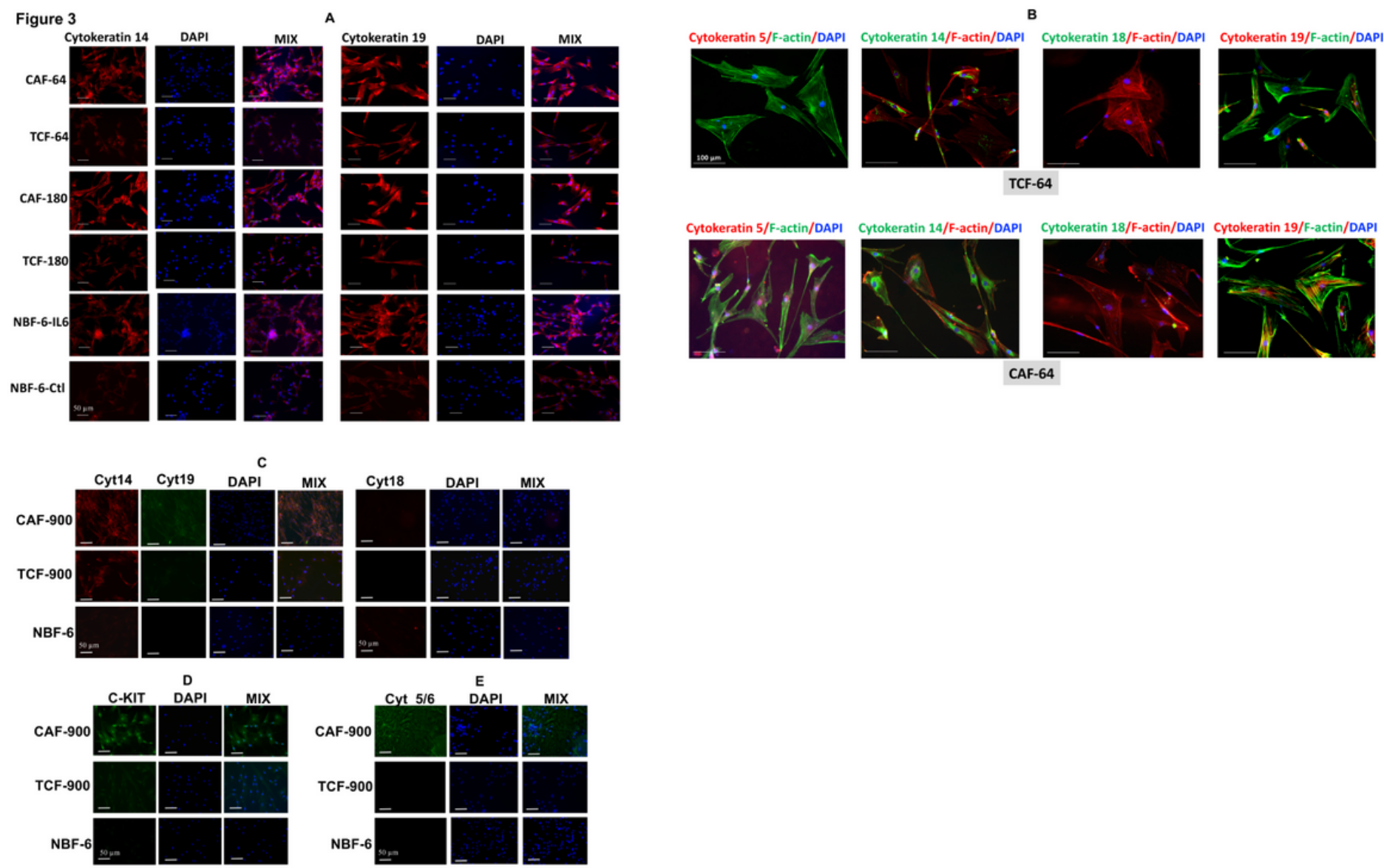

\section{Figure 3}

Active CAFs express myoepithelial markers. A, B and C. Representative images of immunofluorescence staining in the indicated breast fibroblasts using various luminal and myoepithelial markers. 
Figure 4

A

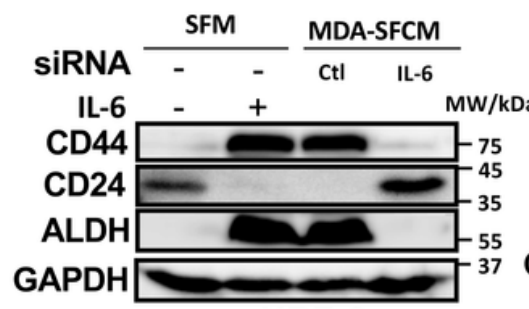

NBF-6
B

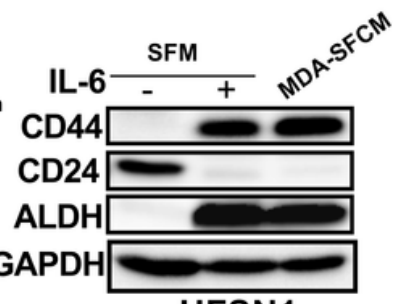

HFSN1
C

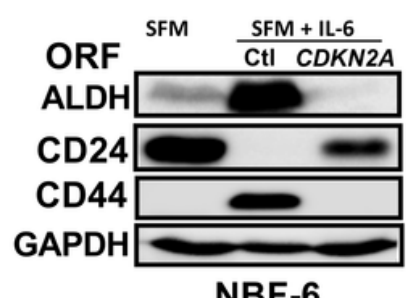

NBF-6
D

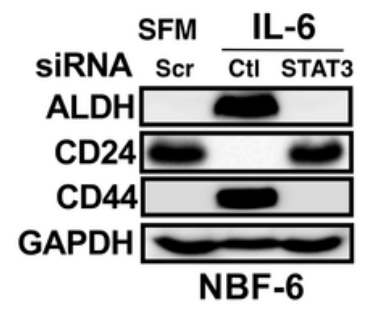

\section{Figure 4}

IL-6 induces stemness features in breast fibroblasts in a STAT3- and p16-dependent manner. (A-D), Whole cell lysates were prepared from the indicated cells and were treated as shown, and then immunoblotting analysis was performed using antibodies against the indicated proteins. 
Figure 5

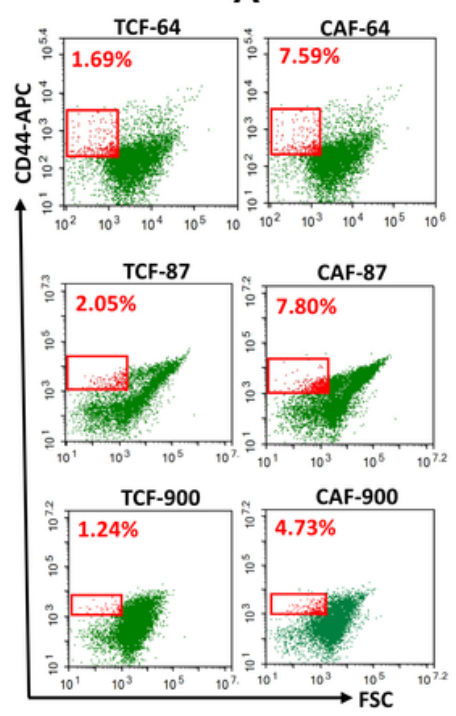

B

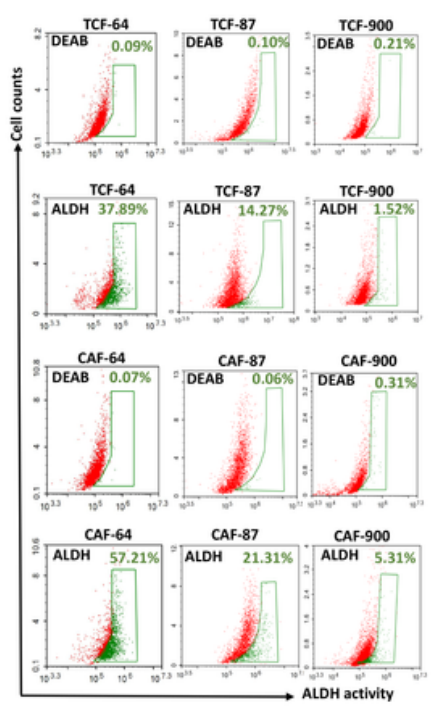

C

$\begin{array}{lllllllllllll}\text { Patients: } & 64 & 84 & 87 & 114 & 118 & 153 & 180 & 169 & 346 & 900 & 69 & 148\end{array}$ Cells: TCF CAF TCF CAF TCF CAF TCF CAF TCF CAF TCF CAF TCF CAF TCF CAF TCF CAF TCF CAF TCF CAF TCF CAF
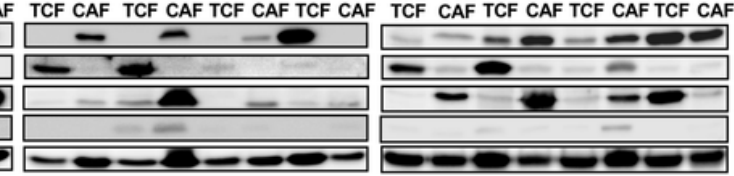

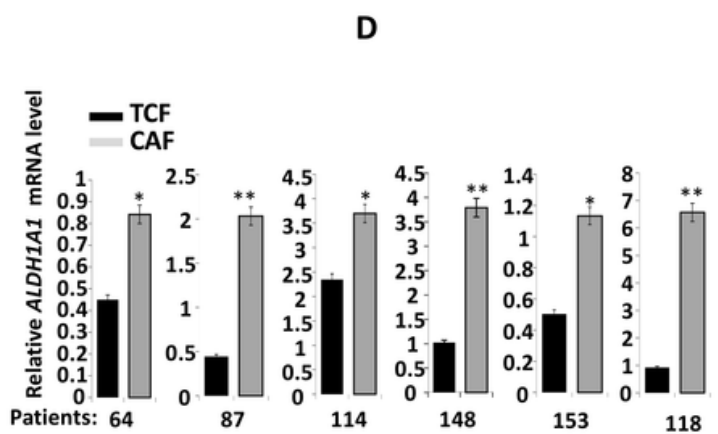

\section{Figure 5}

CAF cells from breast cancer tissues exhibit stemness features. A, Flow cytometric analysis of CD44 expression in the indicated CAF and their corresponding TCF cells. B, ALDH activity was determined by the Aldefluor assay for the indicated cells using flow cytometry. C, Cell lysates were prepared from the indicated CAF/TCF pairs, and then were used for immunoblotting analysis using antibodies against the indicated proteins. D, Total RNA was extracted from the indicated cells, and then the mRNA levels of the indicated genes were determined by qRT-PCR. Error bars indicate mean $\pm S D,(n=3)$. *: $p \leq 0.05 ; * *: p \leq$ 0.01 . 
Figure 6

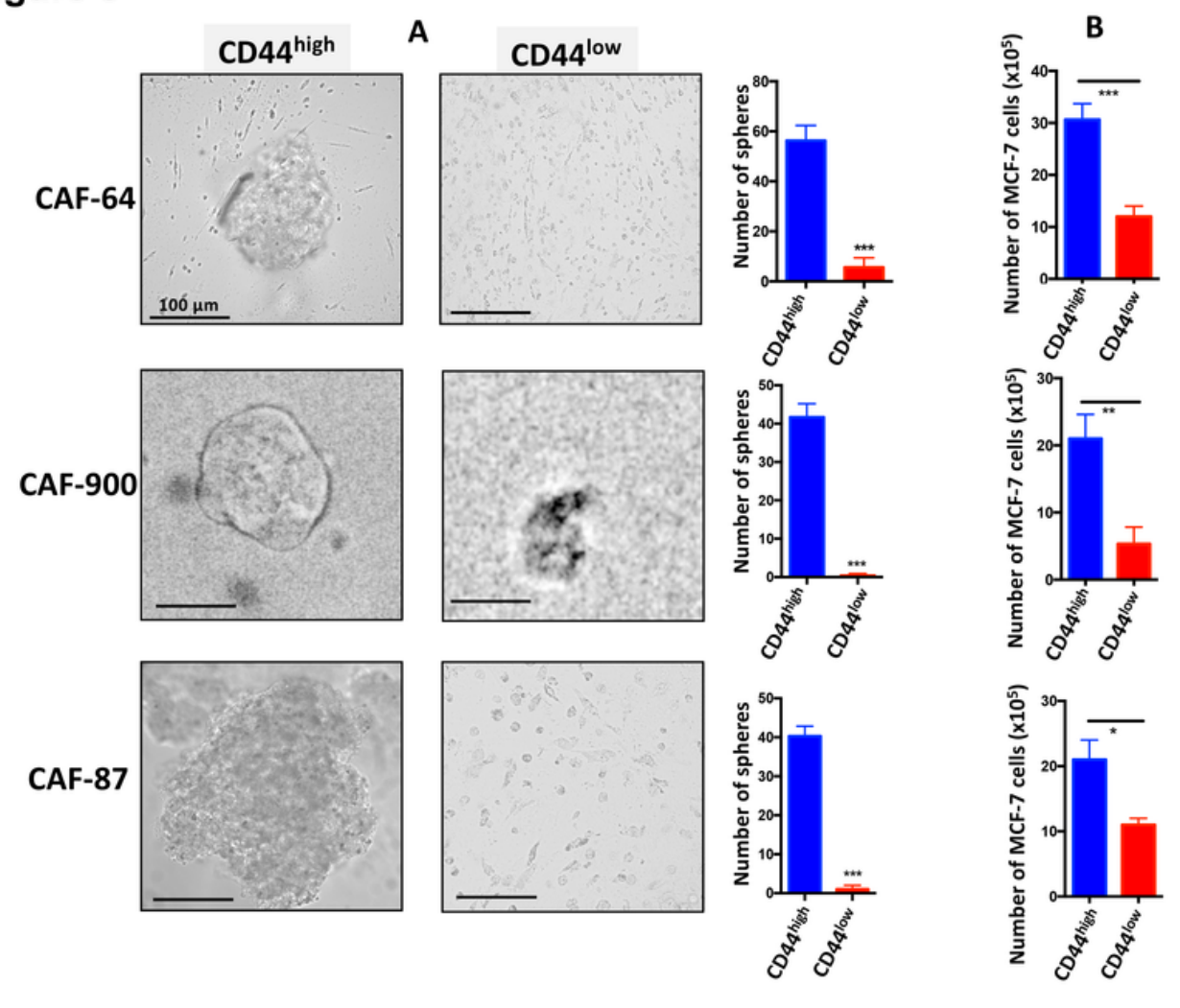

Figure 6

CD44-positive CAF cells form mammospheres and promote breast cancer cells growth. A, Representative images of spheres formed from CAF sorted CD44high/CD44low cells and growth in ultralow attachment stem cells culture conditions. The number of spheres was determined and was plotted as bar graphs. B, Breast cancer MCF-7 cells were co-cultured CD44high/CD44low CAF cells for $24 \mathrm{hr}$, and then cells were counted and plotted as bar graphs. Three independent experiments were conducted for each CAF cells. 